\title{
Control of Action Potential Timing by Intrinsic Subthreshold Oscillations in Olfactory Bulb Output Neurons
}

\author{
David Desmaisons, Jean-Didier Vincent, and Pierre-Marie Lledo \\ Centre National de la Recherche Scientifique, Institut Alfred Fessard, 91198 Gif-sur-Yvette Cedex, France
}

Rhythmic patterns of neuronal activity have been found at multiple levels of various sensory systems. In the olfactory bulb or the antennal lobe, oscillatory activity exhibits a broad range of frequencies and has been proposed to encode sensory information. However, the neural mechanisms underlying these oscillations are unknown. Bulbar oscillations might be an emergent network property arising from neuronal interactions and/or resulting from intrinsic oscillations in individual neurons. Here we show that mitral cells (output neurons of the olfactory bulb) display subthreshold oscillations of their membrane potential. These oscillations are mediated by tetrodotoxin-sensitive sodium currents and range in frequency from 10 to $50 \mathrm{~Hz}$ as a function of resting membrane potential. Because the voltage dependency of oscillation frequency was found to be similar to that for action potential generation, we studied how subthreshold oscillations could influence the timing of action potentials elicited by synaptic inputs. Indeed, we found that subthreshold oscillatory activity can trigger the precise occurrence of action potentials generated in response to EPSPs. Furthermore, IPSPs were found to set the phase of subthreshold oscillations and can lead to "rebound" spikes with a constant latency.

Because intrinsic oscillations of membrane potential enable very precise temporal control of neuronal firing, we propose that these oscillations provide an effective means to synchronize mitral cell subpopulations during the processing of olfactory information.

Key words: mitral cells; sodium current; synchronization; timing device; inhibitory interneurons; olfactory processing
Determining how neurons transform synaptic inputs into spike trains is a necessary step toward understanding information processing in sensory systems (Rieke et al., 1997). The key question is what temporal pattern of spike trains are detected and integrated by postsynaptic neurons. One might consider that a sequence of action potentials contains information either based on the average number of spikes per unit time (rate coding) or, alternatively by the precise timing of individual action potentials (temporal coding): a slow time-scale integration is thought to underlie rate coding mechanisms (Parker and Newsome, 1998), whereas fast time-scale integration is thought to involve coincidence detection in which the precise timing of spikes carries information (for review, see König et al., 1996). Of course, the dichotomy between rate and temporal coding may not be so distinct because the brain might use a combination of these coding systems (Riehle et al., 1997).

Synaptic integration and transformation into spikes depends on voltage-gated ionic conductances that influence both the resting membrane potential and the firing properties of neurons (Hille, 1992). We now know that ionic conductances provide the basis for numerous nonlinear operations, and thus endow neurons with much greater information processing capacities than was previously thought (Spencer and Kandel, 1961; Stuart and Sakmann, 1995; Haag and Borst, 1996). This nonlinearity allows neurons to

\footnotetext{
Received July 12, 1999; revised Aug. 30, 1999; accepted Sept. 28, 1999.

This work was supported by the Centre National de la Recherche Scientifique, the Institut Universitaire de France, and a grant from the Direction des Recherches et Etudes Techniques to D.D. We are grateful to G. Sadoc for help with the analysis and acquisition software. We thank G. M. Shepherd and the members of our laboratory for comments on this manuscript.

Correspondence should be addressed to Dr. Pierre-Marie Lledo, Centre National de la Recherche Scientifique, Institut Alfred Fessard, Avenue de la Terrasse, 91198 Gif-sur-Yvette Cedex, France. E-mail: lledo@iaf.cnrs-gif.fr.

Copyright (C) 1999 Society for Neuroscience 0270-6474/99/1910727-11\$05.00/0
}

integrate synaptic events in a very precise temporal fashion (Larkum et al., 1999), with a temporal fidelity on the order of milliseconds. Voltage-gated conductances can also control the timing of action potentials through the generation of membrane potential oscillations (for review, see Llinás, 1988). Indeed, the interaction between these oscillations and synaptic inputs can provide an effective means to synchronize neurons in an oscillatory assembly (for review, see Connors and Amitai, 1997; Ritz and Sejnowski, 1997). This phenomenon has been reported in various brain structures such as the neocortex (Silva et al., 1991), hippocampus (Cobb et al., 1995), and thalamus (von Krosigk et al., 1993) and may be essential for encoding sensory information (Gray, 1994).

The present study focuses on membrane potential oscillations in output neurons of the main olfactory bulb (mitral cells) and the temporal relationship between these oscillations and action potentials. Temporal coding has been proposed to play an important role in olfactory processing (Tank et al., 1994; Laurent, 1996a). For example, electroencephalogram (EEG) and local field potential (LFP) recordings in the olfactory bulb have revealed network oscillations in a wide range of animals: Limax (Gelperin and Tank, 1990), locust (Laurent et al., 1996), fish (Satou and Ueda, 1978), frog (Delaney and Hall, 1996), and insectivore (Adrian, 1942). This oscillatory activity results from neuronal synchronization (Laurent and Davidowitz, 1994; for review, see Laurent, 1996a,b). In the honey bee antennal lobe (the analog of mammalian olfactory bulb), data suggests that neuronal synchronization may be necessary for neural processing because pharmacologically induced desynchronization of output neurons results in olfactory discrimination deficits (Stopfer et al., 1997).

Here we report that intrinsic oscillations act as a timing device for the integration of postsynaptic potentials into spikes. We propose that interactions between the intrinsic properties of 
mitral cells and their synaptic inputs can efficiently synchronize the activity of multiple mitral cells. This mechanism may allow the representation of odors by temporally distributed ensembles of coherently firing mitral cells.

\section{MATERIALS AND METHODS}

Slice preparation. Experiments were performed on olfactory bulb slices from 4- to 6-week-old Wistar rats. Animals were anesthetized by intraperitoneal injection of pentobarbital $(100 \mu \mathrm{l})$ and then killed by decapitation. The olfactory bulbs were rapidly removed and immediately placed in a standard $4^{\circ} \mathrm{C}$ artificial CSF (ACSF) solution in which $\mathrm{NaCl}$ was replaced with sucrose (the osmolarity was maintained to $310 \mathrm{mOsm}$ ). The standard ACSF contained (in $\mathrm{mM}$ ): $124 \mathrm{NaCl}, 3 \mathrm{KCl}$ (unless otherwise indicated), $2 \mathrm{CaCl}_{2}, 1.3 \mathrm{MgCl}_{2}, 25 \mathrm{NaHCO}_{3}, 1.25 \mathrm{NaH}_{2} \mathrm{PO}_{4}$, and 10 D-glucose, $\mathrm{pH} 7.3$, when bubbled with $95 \% \mathrm{O}_{2}$ and $5 \% \mathrm{CO}_{2}$. Horizontal slices $(400 \mu \mathrm{m})$ were cut on a vibrating microslicer (Vibratome 1000; Ted Pella, St. Louis, MO), incubated in normal oxygenated ACSF at $32^{\circ} \mathrm{C}$ for $\sim 60 \mathrm{~min}$, and then maintained at room temperature $\left(20-22^{\circ} \mathrm{C}\right)$ except when indicated. Individual slices were then transferred to a submergedslice recording chamber where they were superfused with oxygenated ACSF at a rate of $1-2 \mathrm{ml} / \mathrm{min}$. The horizontal plane was chosen because its allows the best orientation for preserving mitral cells with intact primary and secondary dendrites.

Electrophysiological recordings. Extracellular recordings were performed using glass microelectrodes containing $1 \mathrm{M} \mathrm{NaCl}$ and amplified using a DAM-80 (World Precision Instruments, Hertfordshire, UK) differential amplifier. Intracellular recordings were performed with borosilicate glass microelectrodes (Clark Electromedical Instruments, Reading, UK) pulled with the Flaming-Brown microelectrode puller. In some experiments, intracellular recording electrodes were back-filled with $2 \%$ biocytin in $2 \mathrm{M}$ potassium acetate for morphological confirmation. Intracellular current-clamp recordings (electrode resistances ranged between 120 and $140 \mathrm{M} \Omega$ ) were performed with an Axoclamp-2A (Axon Instruments, Foster City, CA) amplifier, and, during experiments, the bridge balance was continuously monitored. To evoke synaptic responses, stimuli (100 $\mu \mathrm{sec}$ duration) were delivered through fine bipolar tungsten electrodes placed in the olfactory nerve layer (ONL), in the external plexiform layer (EPL), or alternately in both layers. Membrane potentials were filtered ( $3 \mathrm{kHz}$ low-pass), collected on-line via the Digidata interface (Axon Instruments) on an IBM-compatible computer, and stored on videotape for later analysis.

Neurons were identified by antidromic stimulation of the lateral olfactory tract, orthodromic stimulation of the olfactory nerve, by their location in the mitral cell layer, and occasionally by biocytin injection. Only those having stable membrane potentials more negative than -50 $\mathrm{mV}$, input resistances $>80 \mathrm{M} \Omega$, and action potentials overshooting $0 \mathrm{mV}$ were retained for analysis. At the end of each experiment, possible changes in electrode tip potential were controlled by measuring the DC offset of the electrode in the bathing medium, and the value of the measured membrane potential was corrected accordingly. Changes in electrode tip potential were usually $<3 \mathrm{mV}$. The current injected to produce oscillations of the membrane potential was a sine wave:

$$
I(t)=\mathrm{I}_{\max } \cdot \cos (2 \pi t / T),
$$

where $\mathrm{T}$ is the period (50 msec for all the experiments), and $\mathrm{I}_{\max }$ the maximal current that was adjusted on-line. The current injected to mimic EPSP was given by the equation:

$$
I(t)=\mathrm{I}_{\max } \cdot\left(1-e^{-\mathrm{t} / \tau \mathrm{r}}\right) \times e^{-\mathrm{t} / \tau \mathrm{d}},
$$

where $\tau_{\mathrm{r}}$ is the rise time, $\tau_{\mathrm{d}}$ is the decay time, and $\mathrm{I}_{\max }$ is the maximum of the current, all three values of which were controlled on-line.

Solutions and drugs. Pharmacologically active substances were applied in the bathing medium using a gravity-driven perfusion system. To block EPSPs, mitral cells were recorded in the presence of a nonspecific ionotropic glutamate receptor antagonist, kynurenic acid $(5-10 \mathrm{mM})$ or in the presence of the NMDA receptor antagonist D,L-2-amino-5phosphonopentanoic acid (D,L-APV; $100 \mu \mathrm{M})$ and the AMPA receptor antagonist 6-cyano-7-nitroquinoxaline-2,3-dione (CNQX; $10 \mu \mathrm{M})$. To block IPSPs mediated by the activation of $\mathrm{GABA}_{\mathrm{A}}$ receptors, cells were bathed with either a $\mathrm{GABA}_{\mathrm{A}}$ receptor antagonist $(20-40 \mu \mathrm{M}$ bicuculline or $20 \mu \mathrm{M}$ SR 95531) or a chloride channel blocker (100 $\mu \mathrm{M}$ picrotoxin). CNQX and SR 95531 were obtained from Research Biochemicals Inter-
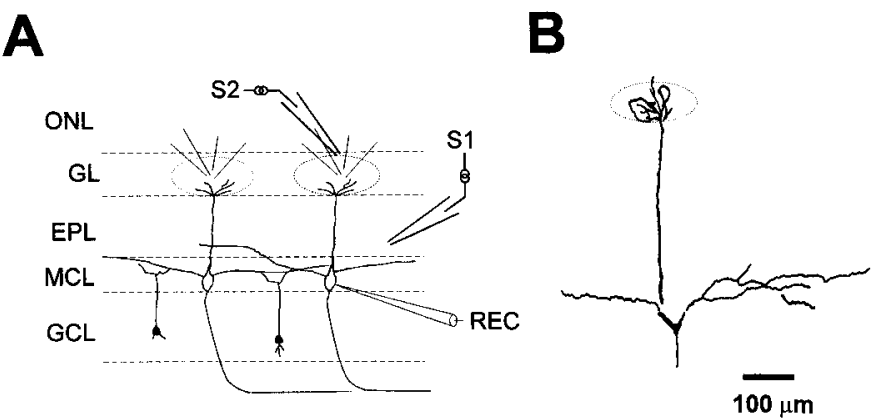

Figure 1. The horizontal rat olfactory bulb slice preparation. A, Experimental arrangement for bipolar stimulating electrodes $(S 1$ and $S 2)$ and the recording electrode $(R E C)$ in a schematic representation of the main olfactory bulb. $O N L$, Olfactory nerve layer; $G L$, glomerular layer; $E P L$, external plexiform layer; $M C L$, mitral cell body layer; $G C L$, granule cell layer. $B$, Camera lucida drawing of a mitral cell labeled intracellularly with biocytin.

national (Natick, MA), D,L-APV from Tocris (Illkirch, France), and all other drugs and salts from Sigma (Strasbourg, France).

Data analysis. Both on- and off-line analyses were performed with Acquis1 software (Gérard Sadoc, Centre National de la Recherche Scientifique-Agence Nationale pour la Valorisation de la Recherche, Paris, France). Mitral cell membrane potential was taken as the average potential during a period without spikes (but which included subthreshold oscillations). Because fast Fourier transformations (FFTs) and autocorrelation of the membrane voltage gave similar results, we used FFT to analyze the rhythmic nature of the subthreshold oscillations and to calculate their dominant frequency. Oscillations were triggered by detecting local maxima of the membrane potential. To characterize the relationship between oscillation phases and spike latency, we arbitrarily attributed the phase value 0 to stimulations performed at a time corresponding to the peak of an oscillation and giving rise to a spike with a latency longer than $25 \mathrm{msec}$. Similarly, we attributed the phase value $2 \pi$ to a stimulation that triggers spikes with a latency longer than $25 \mathrm{msec}$. Such an operation does not bias the results because analyzing data without these two values $(0$ and $2 \pi)$ gave similar results (i.e., dependency between oscillation phase and latency, $p<0.005$; slopes of linear regressions, -6.4 vs $-8.7 \mathrm{msec}$; and $y$-intercept values, 35.7 vs $40.2 \mathrm{msec}$, respectively). Average cross-correlations (ACC) were used to evaluate the precise timing of subthreshold oscillations after a triggering event. These were calculated by averaging the cross-correlations obtained from all pairs of traces measured in different trials, and were expressed according to the formula:

$$
A \mathrm{cc}=\sum_{i \neq j} C c(i, j) /\left(n^{2}-n\right),
$$

where $C c(i, j)$ is the cross-correlation between the $i^{\text {th }}$ and the $j^{\text {th }}$ trial of the triggering event (maximal value normalized to 1 ), and $n$ the number of trials. Spontaneous IPSPs were detected using a negative threshold on the slope of membrane voltage and manually accepted or refused. The decay time constant $(\tau)$ of synaptic events was calculated by fitting the average membrane potential (at least ten trials) with an exponential function:

$$
V(t)=\mathrm{V}_{0} \cdot e^{-\mathrm{t} / \tau} .
$$

All the peristimulus-time, autocorrelation, and cross-correlation histograms were computed using methods previously described (Perkel et al., 1967). Unless otherwise indicated, the bin width of all histograms is 10 msec. Statistical significance of differences between means was assessed using a Student's $t$ test. Kolmogorov-Smirnov statistics were also used to determine whether there were statistically significant changes between different groups, and the level of significance was set at $p<0.05$. Unless otherwise indicated, data are expressed as mean \pm SEM.

\section{RESULTS}

During the course of this study, intracellular recordings were performed on 85 mitral cells (see Fig. $1 A$ for the experimental 


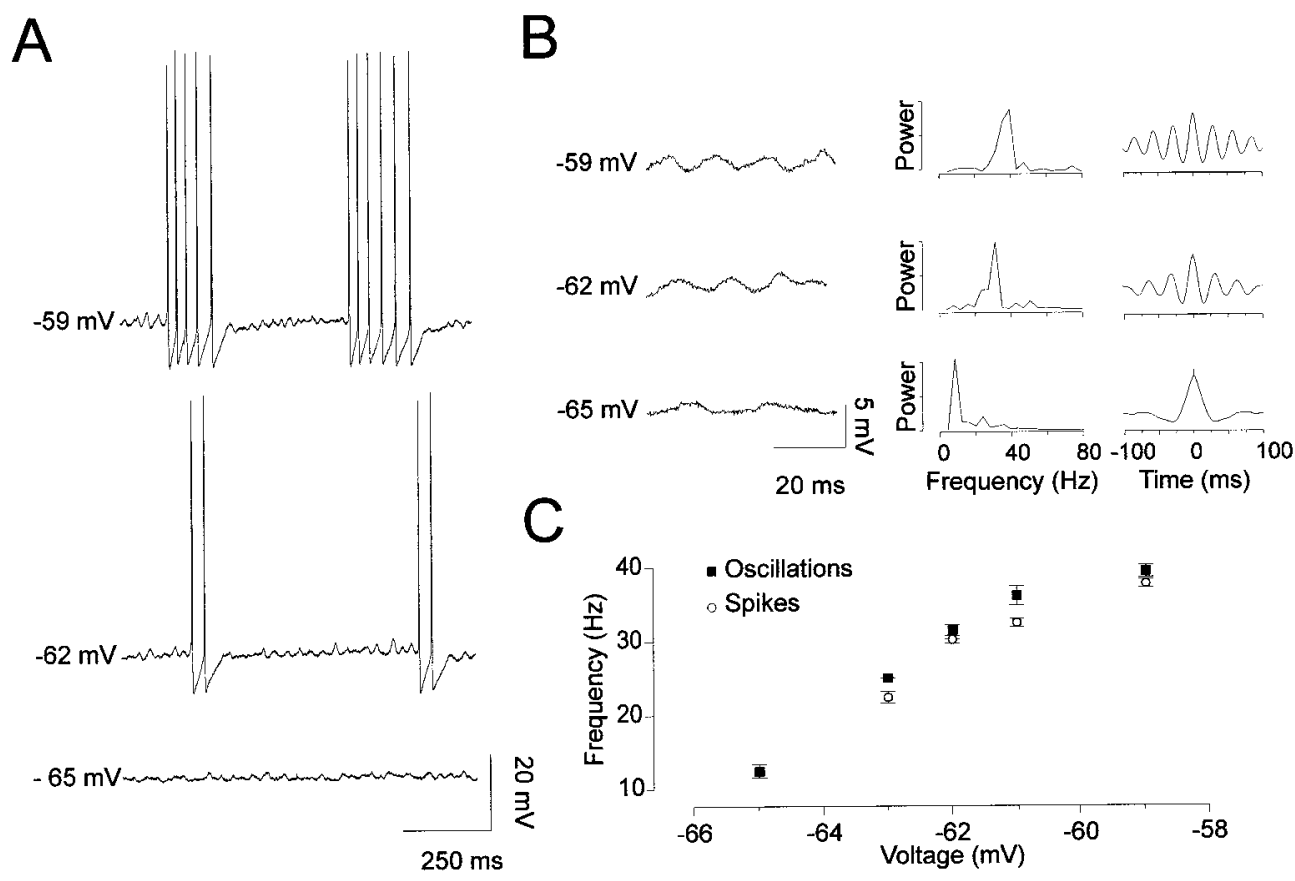

Figure 2. Subthreshold membrane potential oscillations are voltage-dependent. $A$, Increasing depolarization by injection of a constant current triggers clustered action potentials interspersed by membrane potential oscillations (top two traces). Slow oscillations are observed at more hyperpolarized membrane potentials (bottom trace). B, Power spectrum (arbitrary units) reveals the dominant frequency of these oscillations (middle panel), whereas the autocorrelation indicates their rhythmic nature (right panel). Left, Expanded view of the oscillations at three different potentials used for the corresponding graphs (same cell as in $A$ ). C, Voltage dependency of oscillation frequency ( filled symbols) and spike frequency (open symbols) from the same cell. Symbols represent the mean, and error bars indicate SEM. The spike frequency is calculated as the inverse of the mean of the first interspike interval within a cluster.

setup and Fig. $1 B$ for mitral cell morphology). With pipettes containing a $\mathrm{K}^{+}$-based intracellular solution, these neurons had a mean resting membrane potential of $-62.7 \pm 1.7 \mathrm{mV}$ (range, -71 to $-51 \mathrm{mV} ; n=12$ ), a mean spike width of $1.98 \pm 0.10 \mathrm{msec}$ when measured at half-maximum amplitude $(n=19)$, a mean time constant of $19 \pm 3 \mathrm{msec}$ (range, $7-40 \mathrm{msec} ; n=19$ ), and a mean membrane input resistance of $128 \pm 10 \mathrm{M} \Omega$ (range, 88-280 $\mathrm{M} \Omega$; $n=19)$. Successfully impaled neurons could be held up to $7 \mathrm{hr}$ without any change in these membrane properties.

\section{The frequency of subthreshold oscillations is voltage-dependent}

In all mitral cells, spontaneous subthreshold oscillations in membrane potential were observed at resting membrane potential (Fig. 2A,B). These oscillations were not dependent on synaptic input because they persisted in the presence of the selective $\mathrm{GABA}_{\mathrm{A}}$ antagonist bicuculline (40 $\left.\mu \mathrm{M} ; n=16\right)$, the broadspectrum ionotropic glutamatergic antagonist kynurenate (10 mM; $n=7)$, or a mixture of both $(n=6)$. They were observed at a wide spectrum of membrane potentials, and their amplitude ranged from 2.1 to $5.7 \mathrm{mV}$ (a mean of $3.7 \pm 0.1 \mathrm{mV} ; n=51$ ). Figure 2, $A$ and $B$ displays the voltage traces of a mitral cell that was depolarized by steady current injections of increasing amplitude. Subthreshold oscillations first appeared in the absence of action potentials (approximately $-67 \mathrm{mV}$ ). After further depolarization, clear rhythmic deflections of the membrane potential were detected whose amplitude was sufficient to reach the action potential threshold $(-65 \mathrm{mV})$. The firing pattern at this potential was characteristic of mitral cells recorded in slice preparations: clusters of spikes interspersed with long period of subthreshold oscillations (Fig. 2A, top trace; see also Chen and Shepherd, 1997, their Fig. 2B). To characterize the voltage dependency of these oscillations, we used FFTs and autocorrelations to calculate their frequencies. As shown in Figure 2, $B$ and $C$, the oscillation frequency was found to be $\sim 10 \mathrm{~Hz}$ near their threshold and rose to $20 \mathrm{~Hz}$ at the spike threshold. Further depolarization of the cell progressively increased the frequency of subthreshold oscillations up to $40 \mathrm{~Hz}$ at $-59 \mathrm{mV}$ (Fig. 2B, top graphs). All cells analyzed showed similar voltage dependency $(n=5)$.

We found that across potentials, the frequency of oscillations and the instantaneous spike frequency (measured as the inverse of the mean of the first interval interspike within a cluster) were highly correlated. As illustrated in Figure $2 C$, the frequency of subthreshold oscillations and spikes exhibited almost parallel increases as a function of membrane potential ( $n=4$ of 4$)$. This close relationship was further demonstrated by a linear regression analysis of oscillation frequency plotted against spike frequency [Fig. 3A; mean slope linear regression $(k) ; 1.05 \pm 0.14(n=4)$, with all cells presenting $r>0.97]$.

To further characterize the relationship between subthreshold oscillations and firing activity, the temporal relationship between spikes and oscillations was investigated. As previously described in other preparations (Llinás et al., 1991; Gutfreund et al., 1995), we found that action potentials were synchronized with the peak of the subthreshold oscillations $(n=5$ of 5$)$. Figure $3 B a$ shows spike-triggered voltage traces illustrating that spikes and oscillations are phase-locked. The rising slopes of these two events are superimposable, and the spike initiation point is always during the rising phase. Similarly, calculating the probability that the cell will spike after one oscillation clearly shows that mitral cell spiking is triggered by these oscillations (Fig. $3 B b$ ). From the 


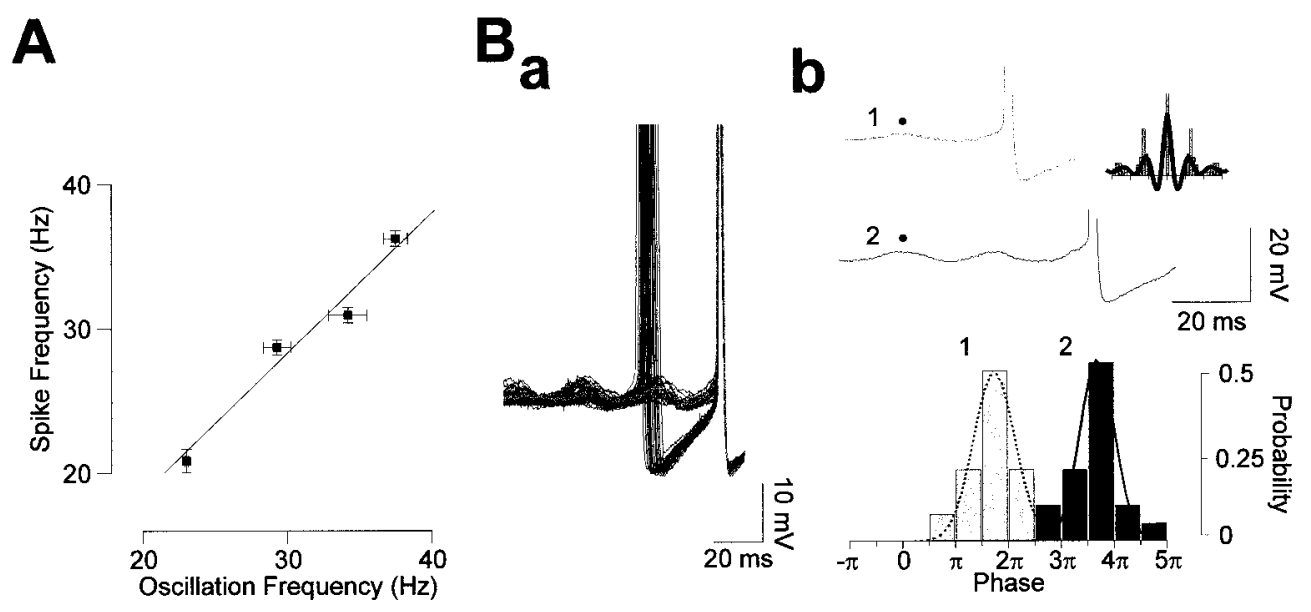

Figure 3. Relationship between subthreshold oscillations and spikes. $A$, The average oscillation frequency is plotted as a function of the mean spike frequency with a linear regression $(k=0.997)$. Each symbol represents the mean \pm SEM. $B$, Temporal correlation between spikes and oscillations. $a$, Twenty superimposed spike-triggered traces and corresponding average of traces with one spike. $b$, Phase relationship between spikes and subthreshold potential oscillations. Traces were triggered on the last (trace 1) or on the penultimate oscillation (trace 2) before spike emission to establish a probability histogram of spike emission as a function of the oscillation phase. Results were fitted with Gaussian functions. Note the tendency for the cell to fire with a small phase advance. Inset, Autocorrelation of the subthreshold oscillations preceding a spike (continuous line), and autocorellogram of spikes (histogram) for the same potential, illustrating similar frequencies for the two events (spikes are clipped for clarity).

A

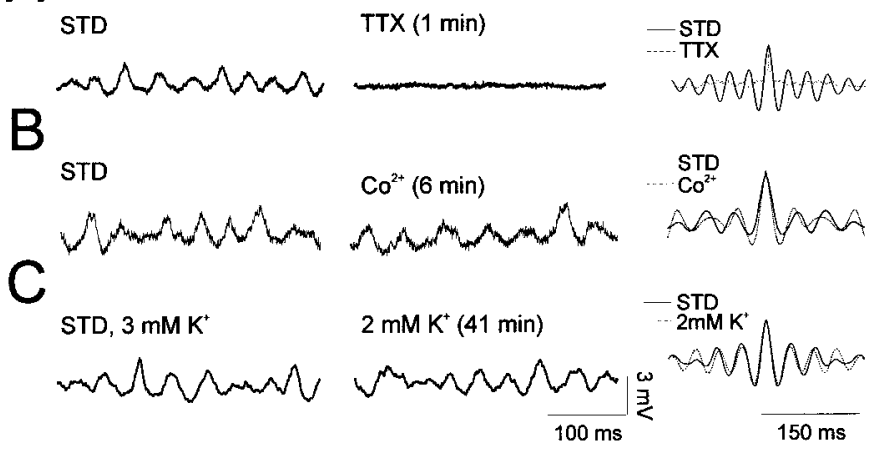

Figure 4. Pharmacology of subthreshold oscillations. Control traces recorded in standard medium (left) and in external medium containing either $1 \mu \mathrm{M}$ tetrodotoxin $(A)$, cobalt substituted for calcium $(B)$, or with a lower $\mathrm{KCl}$ concentration $(C)$, after a perfusion time, indicated in parentheses. Right panels, Corresponding autocorrelations. Note that oscillations were only abolished after bath application of TTX, whereas calcium withdrawal or lowering of external potassium affected neither their frequency nor their amplitude $\left(\mathrm{V}_{\text {hold }}=-62 \mathrm{mV}\right.$ for all traces).

probability histograms for spiking shown in Figure $3 B b$, it can be seen that the generation of a spike coincides with the peak of the following oscillation with, however, a small phase advance. This anticipation can also be seen by close inspection of the superimposed voltage traces displayed in Figure $3 B a$.

\section{lonic conductance underlying the subthreshold oscillations}

We next investigated the potential role of ionic conductances in the generation of membrane potential oscillations (Fig. 4). First, the broad-spectrum sodium channel blocker tetrodotoxin (TTX; $1 \mu \mathrm{M})$, completely blocked these membrane potential oscillations (Fig. $4 A ; n=4$ of 4 ). Then, to determine whether calcium also could be involved, it was removed from the extracellular medium and substituted with cobalt $(2 \mathrm{~mm})$. We were not able to detect any changes in either the frequency or the amplitude of the oscillations ( $n=4$ of 4 ), even after a perfusion time of $>12 \mathrm{~min}$
(Fig. 4B), when synaptic transmission was blocked (data not shown). Finally, the potential role of potassium conductances was first addressed with the potassium channel blocker tetraethylammonium (TEA). When $10 \mathrm{~mm}$ TEA was added to the perfusate, input membrane resistances and firing activity changed dramatically (data not shown), so evaluating their participation in oscillatory activity was meaningless. We thus decided to reduce the external concentration of potassium (from 3 to $2 \mathrm{~mm}$ ) to modify all potassium currents. Lowering extracellular potassium affects AHP amplitude (5 mV more hyperpolarized; data not shown), but no change in subthreshold oscillations amplitude or frequency was observed (Fig. $4 C ; n=4$ of 4 ). From these pharmacological studies, it can be concluded that subthreshold oscillations may result from the activation of a voltage-gated sodium-permeable channel, but neither voltage-gated calcium nor potassium currents seem to be involved.

\section{Spontaneous IPSPs reset the phase of subthreshold oscillations}

In some neurons (18 of 85 neurons), we observed spontaneous synaptic inhibitory events (Fig. $5 \mathrm{~A}$ ) that were completely antagonized by bicuculline $(20-40 \mu \mathrm{M})$ or SR $95531(20 \mu \mathrm{M})$. The mean amplitude of these spontaneous synaptic potentials was $3.2 \pm 0.4 \mathrm{mV}(n=7)$, and they were easily distinguished from oscillations by their kinetics (rise time, $5.1 \pm 0.5 \mathrm{msec}$ vs $22.4 \pm$ $3.2 \mathrm{msec}$ for IPSPs and oscillations, respectively; $n=7$ ). Because it has been reported that GABAergic events can synchronize neuronal activity (Cobb et al., 1995; MacLeod and Laurent, 1996), the temporal relationship between spontaneous IPSPs and membrane potential oscillations was studied. As illustrated in Figure 5, spontaneous IPSPs $(n=7)$ could reset the phase of spontaneous oscillations without affecting their amplitude (Fig. $5 A$ ) or frequency (Fig. $5 C$ ).

Interestingly, this effect induced by spontaneous IPSPs could be mimicked by injecting hyperpolarizing current pulses $(<4$ $\mathrm{mV}$ ) of short duration (10 msec). Hence, as described for spontaneous IPSPs, these current pulses were able to reset the oscil- 


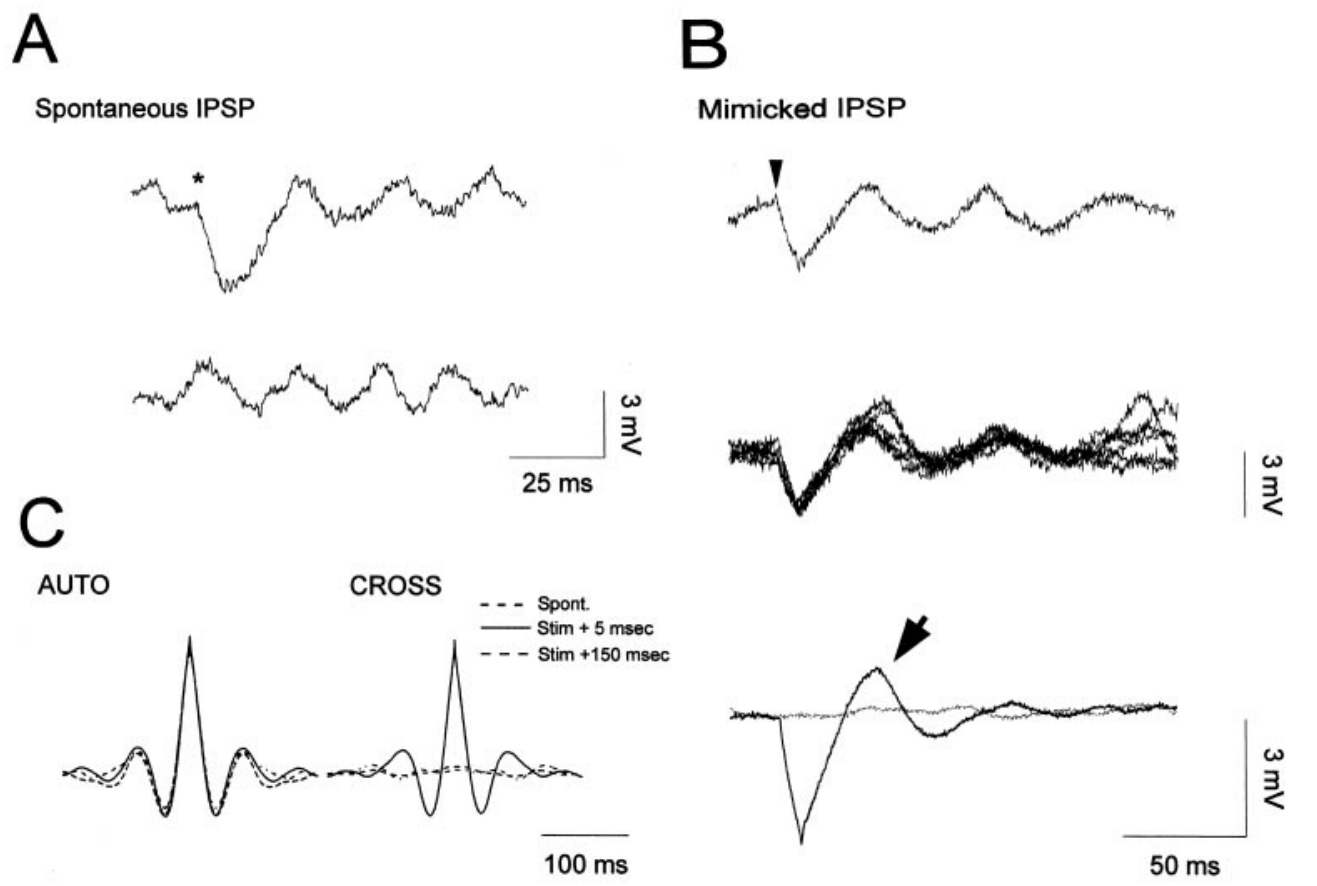

Figure 5. IPSPs or hyperpolarizing pulses reset the phase of the oscillations. $A$, Intrinsic membrane potential oscillations recorded at the same potential in the absence (bottom trace) or the presence (top trace, asterisk) of a spontaneous IPSP. Note that the IPSP only resets the phase of the oscillations without affecting its frequency or amplitude. B, Mitral cell recorded in the presence of bicuculline (40 $\mu \mathrm{M})$ and kynurenate (10 mM). A hyperpolarizing current pulse (arrowhead) is injected to mimic spontaneous IPSPs. From top to bottom, An individual voltage trace showing the triggering of oscillations by a current pulse; six superimposed traces; average of 20 trials compared to the average of traces without pulse. $C$, The hyperpolarizing pulse reset the phase of the oscillations without affecting their frequency. Left, Corresponding averaged autocorrelations without (Spont.), 5 msec (Stim $+5 \mathrm{msec}$ ), or $150 \mathrm{msec}$ after the hyperpolarizing pulse $(\mathrm{Stim}+150 \mathrm{msec})$. No change in frequency can be detected. Right, Averaged cross-correlation obtained from voltage recordings in the same conditions. Results from this correlation can be taken as an index of phase coherence. Note that the subthreshold oscillations are only rephased during $150 \mathrm{msec}$ after the pulse.

lation phase with no effect on the frequency or amplitude $(n=13$; Fig. $5 B$ ), indicating that the reset only results from the IPSPassociated hyperpolarization interacting with intrinsic membrane conductances. To further quantify the effect of these current pulses, we performed autocorrelation analysis (Fig. 5C). First, the averaged autocorrelation of membrane potential measured either, (1) without the pulse, (2) $5 \mathrm{msec}$ after the pulse, and (3) 150 msec after the pulse, demonstrates that the oscillation periods was not modified by the current pulse $(45 \pm 4.8 \mathrm{msec}$ in control conditions and $50 \pm 5.1 \mathrm{msec}$ after the pulse; $n=9 ; p>0.05)$. We then used averaged cross-correlations (ACC) as an index of phase coherence. Indeed, ACC of phased signals resulted in a periodic function similar to the autocorrelation, whereas nonphased signals gave a flat ACC. We found that ACC and autocorrelation were only similar when calculated immediately after the hyperpolarizing pulse, but not $150 \mathrm{msec}$ later, nor without the pulse $(n=10$; Fig. $5 C)$. Hence, the ACC value corresponding to time 0 is significantly different between sweeps with and without pulse $(0.79 \pm 0.05$ vs $0.06 \pm 0.03$, respectively; $p<0.0001)$, indicating a resetting of the oscillation phase after the pulse. Moreover, these ACC showed pronounced multiple peaks that reflect a phasing lasting more than one cycle (corresponding period, $48 \pm$ $5 \mathrm{msec}$ ). Together these results show that small hyperpolarizations can reset the oscillation phase for a period of three cycles. This finding is consistent with the average potential traces collected during the IPSP-mimicking current pulse protocol (Fig. $5 B$, bottom traces).

\section{Spontaneous IPSPs control the timing of mitral cell firing}

For membrane potentials more depolarized than $-65 \mathrm{mV}$, the same hyperpolarizing pulses generated either a single "rebound" action potential or a cluster of spikes $(n=21$; Fig. $6 A)$. Interestingly, evoked or spontaneous IPSPs are also able to produce rebound spikes $(n=11$; Fig. $6 B$ ). Because IPSP amplitudes fluctuated substantially, variable delays in spike initiation could be expected. For example, in the cerebellum, the delay for spike occurrence is proportional to IPSP amplitude (Häusser and Clark, 1997). However, recordings from mitral cells (Fig. 6C) showed no correlation between IPSP slope and spike latency $(p>0.3 ; n=6)$. This result demonstrates that even with a high variability of IPSP amplitude (Fig. 6B,C), inhibitory events were still effective in generating action potentials within a narrow time window. It is noteworthy that this temporal window corresponds to the depolarizing overshoot of subthreshold oscillations after IPSPs, as depicted in Figure 5B (bottom trace, arrow). We further studied the relationship between the spike delay and the holding membrane potential. Figure $7 A$ shows that the spike latency was constant and independent of the membrane potential. This observation held true for membrane potentials ranging from -62 to $-52 \mathrm{mV}(p>0.5 ; n=5)$. Finally, the distribution of spike delays induced either by spontaneous IPSPs or mimicked IPSPs was investigated in the recorded mitral cell population. Interestingly, spike delays generated either by spontaneous GABAergic events (31.1 $\pm 0.6 \mathrm{msec} ; n=11)$ or current-induced hyperpolarizations 
A
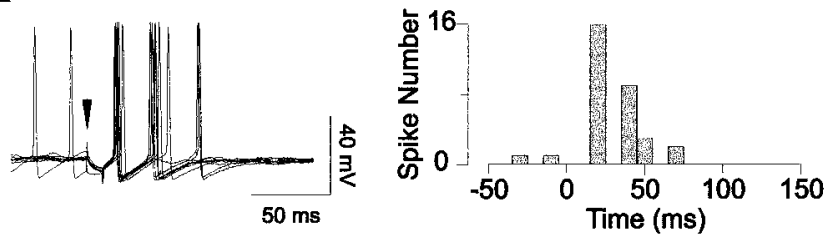

B
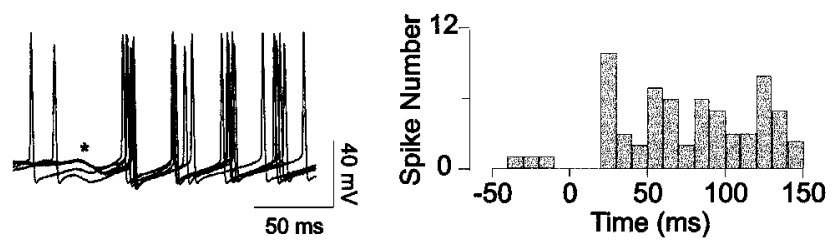

C

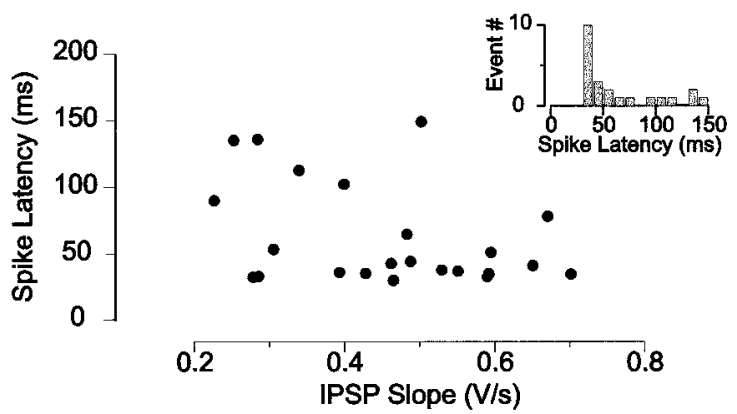

Figure 6. Spontaneous IPSPs or hyperpolarizing current pulses can generate rebound spikes. $A$, A hyperpolarizing current pulse (arrowhead) can elicit a rebound spike. Left, Superimposed sweeps $(n=8)$ illustrating the phasing of spiking after the current pulse. Right, Associated PSTH from all trials $(n=16)$. $B$, Spontaneous IPSPs (asterisk) can also elicit a rebound spike. Five superimposed traces triggered by spontaneous IPSPs (left), and the corresponding PSTH obtained from 23 sweeps (right) showing a marked peak at $30 \mathrm{msec}$ after the spontaneous IPSP. $C$, Relationship between the IPSP slope and the spike delay. Note the absence of any correlation $(r=0.38)$. Inset, Histogram of spike latency.

$(29.2 \pm 0.4 \mathrm{msec} ; n=21)$ were similar (Fig. $7 B ; p>0.05)$. It is also noteworthy that the temporal control for the generation of action potentials was highly uniform because $>85 \%$ of mitral cells generated an action potential within a time window of $10 \mathrm{msec}$ (the distribution of delays ranged from to 33 to $43 \mathrm{msec}$ ).

\section{Subthreshold oscillations are a timing device for synaptic integration}

Having found that IPSPs could synchronize the subthreshold oscillations, we further tested whether the temporal integration of EPSPs into spikes was dependent on this oscillatory activity. Excitatory synaptic responses were evoked by extracellular stimuli applied in the EPL (see Fig. 1 for the location of the stimulating electrode $S 1)$. This stimulation induced short-latency $(<3$ msec), small amplitude $(2.8 \pm 0.5 \mathrm{mV})$, and long duration (halftime decay of $61.8 \pm 10.7 \mathrm{msec}$ ) EPSPs (Fig. $8 A c ; n=12$ ). They were mediated by glutamate receptors because a mixture of D,L-APV $(100 \mu \mathrm{M})$ and CNQX $(10 \mu \mathrm{M} ; n=3)$, or kynurenate (10 mM; $n=4)$ completely blocked these responses. Moreover, bath application of APV alone revealed an AMPA component with a shorter duration $(46.1 \pm 14.6 \mathrm{msec} ; n=5)$.

To test the ability of membrane oscillations to synchronize firing activity, we induced oscillations by sinusoidal current injec-
A

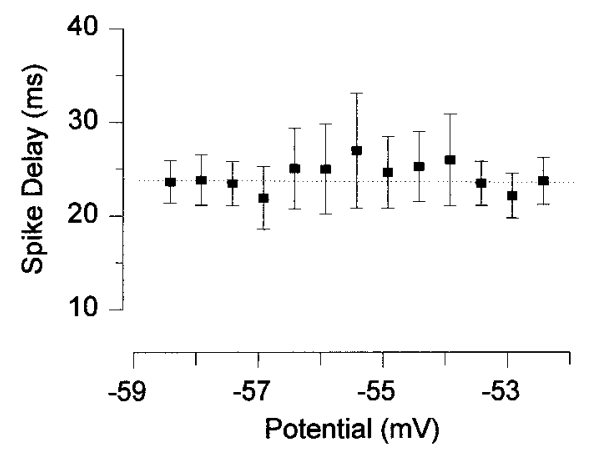

B

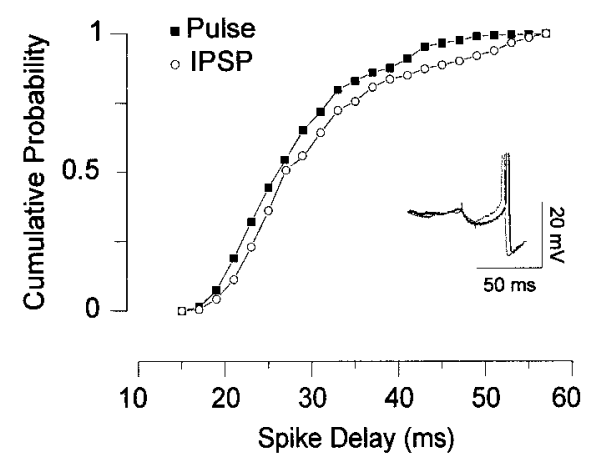

Figure 7. The rebound spike delay is independent from the membrane potential and does not depend on how it is generated. $A$, Mean $\pm \mathrm{SD}$ of the delay between the hyperpolarizing pulse and the spike initiation as a function of the membrane potential for the same cell. Note the absence of correlation $(r=0.08 ; p>0.8)$. $B$, Cumulative probability plots for the spike latency after hyperpolarizing pulses ( filled symbols; $n=21$ cells) or IPSPs (open symbols; $n=8$ cells). The difference between these two populations is not significant $(p>0.05)$. Inset shows two superimposed voltage traces from the same cell illustrating the rebound spike after a current pulse or a spontaneous IPSP.

tion (mean amplitude, $2.7 \pm 0.2 \mathrm{mV}$ ) near spike threshold $(-62.5 \pm 2.5 \mathrm{mV})$ to mimic spontaneous oscillations (Fig. 8Ab). Current-induced oscillations were chosen over endogenous oscillatory activity because they were easier to control. Using this protocol, the relationship between the timing of the EPL stimulation and the spiking activity was studied (Fig. 8B). Although synaptic potentials were triggered at different phases of the sinusoidal waveform, we found that action potentials induced by EPL stimulation $(n=5)$ systematically occurred in a very narrow time window corresponding to the peak of the next oscillation (Fig. $8 B b, B c)$. This time window corresponds to the peak of the following oscillations with a $10 \mathrm{msec}$ jitter (SD, $10.8 \mathrm{msec} ; n=5)$. As a consequence, the delay for generating action potentials was found to depend on the EPL stimulation phase (Fig. 8C). When the delay of spike emission was plotted against the stimulation phase (Fig. $8 B c, 8 C$ ), a clear relationship was found ( $p<0.0001$; $n=5)$. The delay was longer for stimulations occurring during the trough of the oscillation $(\phi=\pi / 2)$ and shorter for stimulations near the peak of the oscillation $(\phi=3 \pi / 2$, for example). To further characterize the temporal relationship between EPSPs and spiking activity, the relationship between the EPSP slope and the stimulation phase was determined after the sine wave responses were subtracted. In all tested cells $(n=3)$, no depen- 

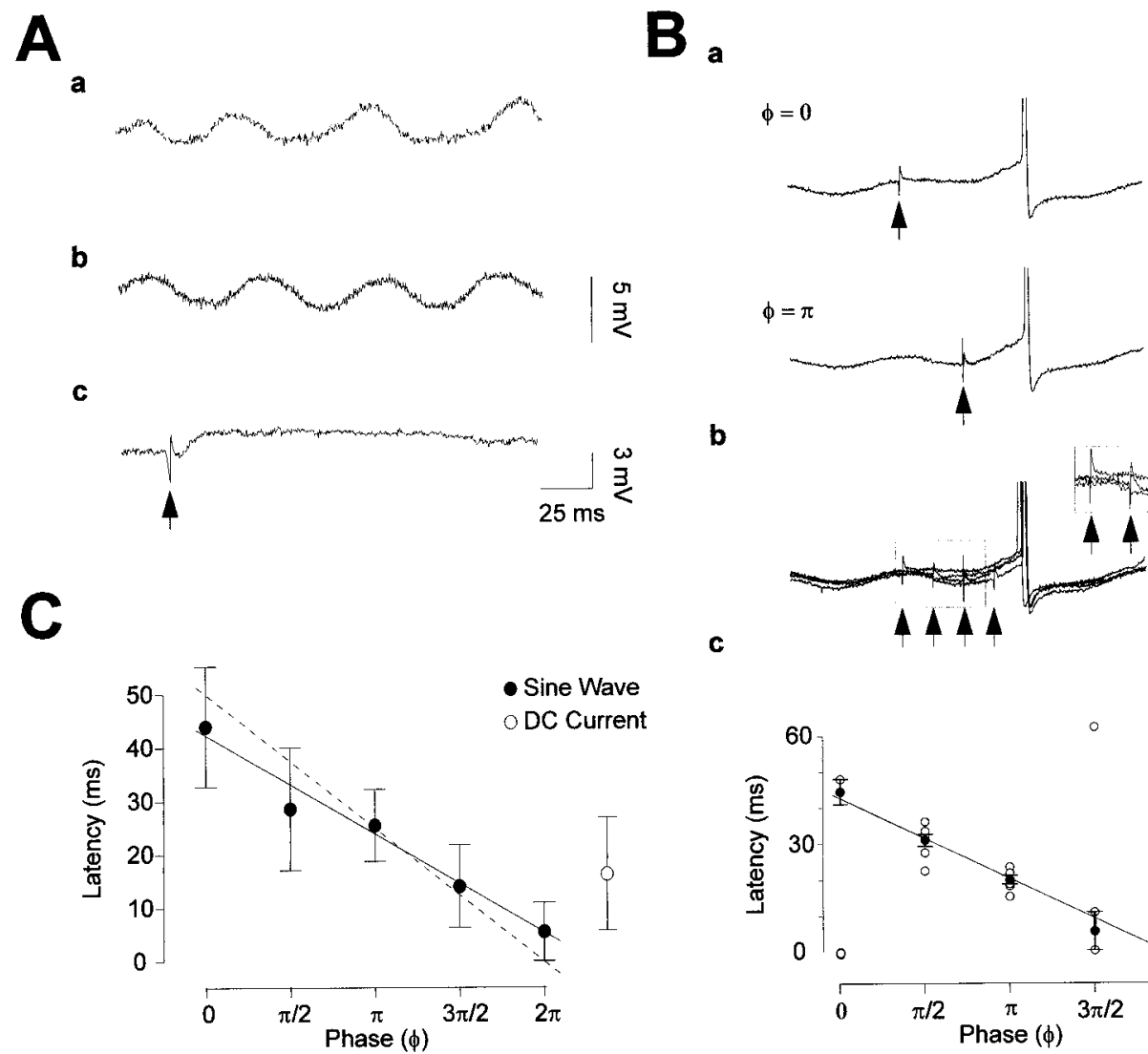

b

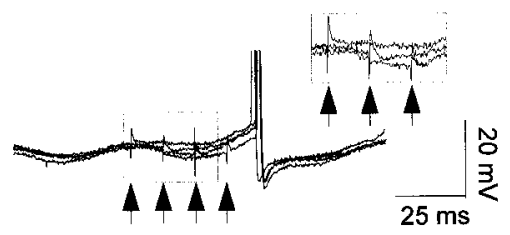

C

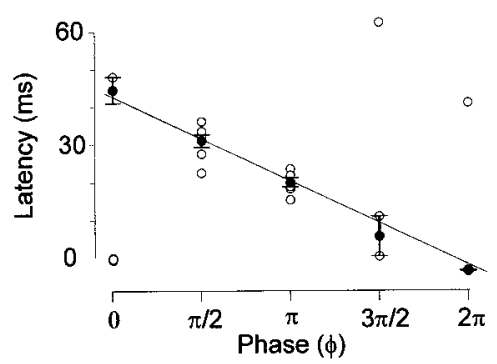

Figure 8. Subthreshold membrane potential oscillations and EPSP integration. $A$, Voltage traces illustrating the experimental protocol. $A a$, A trace showing spontaneous membrane potential oscillations and $(A b)$ sinusoidal current-induced membrane oscillations. $A c$, Averaged evoked EPSP (10 trials) induced by stimulation (arrowhead) in the EPL. $B$, Membrane oscillations act as a timing device for EPSP integration. $B a$, Traces in which the EPL stimulation occurred at the peak (considered as $\phi=0)$ or the trough $(\phi=\pi)$ of the oscillations. Bb, Superimposition of four sweeps differing by $\pi / 2$. Note the constant timing for spike initiation (spikes are truncated). Inset, Expanded view of these traces illustrating the initial EPSPs. Bc, Relationship between the delay for spike initiation and the phase of the stimulation for this cell (open circles) and averaged data \pm SEM ( filled circles) plotted with a linear regression $(r=0.91$; black line $)$. $C$, Summary graph $(n=5$ cells $)$ in which the averaged delay is plotted as a function of the oscillations phase, with corresponding SD ( filled circles). Dashed line, Delay corresponding to a spike occurring at the peak of one oscillation. Open circle, Spike latency for identical holding potentials without induced oscillations ( $D C$ current). Note that for phases exceeding $\pi$ the spike latency variability (quantified by the SD) is smaller in the presence of oscillations than in their absence (see Materials and Methods for more details).

dency was found between these two parameters $(p>0.5)$ or between membrane potentials and EPSP slopes $(p>0.9)$. These observations suggest that voltage-dependent conductances do not participate in the phasing of spikes.

\section{Stimulation of primary afferent axons triggers subthreshold oscillations}

We tried to elucidate a functional role of subthreshold oscillations by electrically stimulating primary afferent axons (see Fig. 1 for the location of the stimulating electrode $S 2)$. A single stimulation of the olfactory nerve $(n=10)$ evoked large EPSPs $(9.1 \pm$ $1.2 \mathrm{mV}$ ) of long duration (mean decay time, $272 \pm 38 \mathrm{msec}$ ). These synaptic responses were mediated by glutamatergic receptors because they were virtually eliminated by bath application of $10 \mathrm{~mm}$ kynurenate $(n=7$; Fig. $9 A a)$. To determine the participation of NMDA and non-NMDA receptors, the amplitude and time decay of synaptic responses were measured in the absence and the presence of APV $(100 \mu \mathrm{M})$ (Fig. $9 \mathrm{Aa} ; n=7)$. The results from 10 experiments performed in standard external solution are summarized in Figure $9 A b$. These experiments revealed that the slow component to the EPSP decay was abolished by APV (mean decay time in APV, $108 \pm 34 \mathrm{msec}$ ), whereas the peak amplitude was relatively unaffected $(6.6 \pm 1 \mathrm{mV})$. Thus, excitatory trans- mission between receptor neurons and mitral cells is mediated by both NMDA and non-NMDA receptors, a finding that is consistent with previous reports (Ennis et al., 1996; Keller et al., 1998).

At resting membrane potentials $(-62.7 \pm 1.7 \mathrm{mV})$, olfactory nerve stimulation produced a long depolarization triggering action potentials ( $n=14$; Fig. 9B). Consequently, mitral cells fired in a rhythmic manner with a clustered spike discharge interspersed with subthreshold membrane potential oscillations (Fig. $9 B a$ ). To confirm that these oscillations were truly intrinsic, an EPSC-like current was injected in the soma of mitral cells recorded in the presence of kynurenate $(10 \mathrm{~mm})$ and bicuculline (40 $\mu \mathrm{M})$. Figure $9 B b$ shows that simulated olfactory nerve impulses give rise to similar clusters of spikes separated by subthreshold oscillations. Identical results were obtained from four different experiments.

The long-lasting response to olfactory nerve stimulation was confirmed with extracellular recordings (data not shown). Most mitral cells were silent at rest $(n=10$ of 15$)$ or fired at a low rate (range, $0.6-9 \mathrm{~Hz} ; n=5$ of 15 ), and they responded to olfactory nerve stimulation with a prolonged period of spiking $(475 \pm 50$ msec; $n=10$ ) compatible with the generation of steady-state depolarizations and subthreshold oscillations shown in our intracellular recordings. Finally, we tested whether IPSPs triggered by 


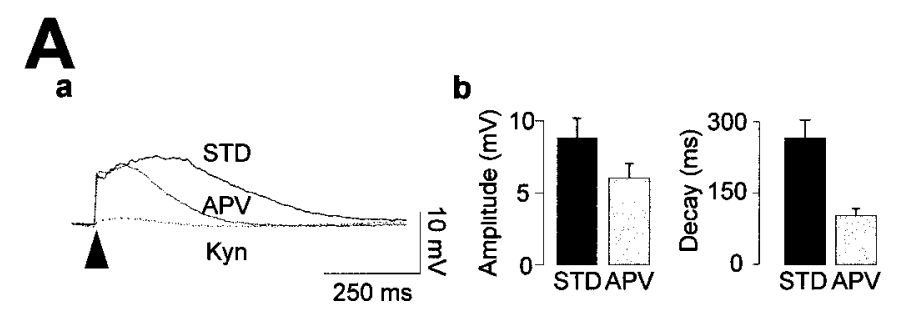

B

\section{O.N. Stim.}
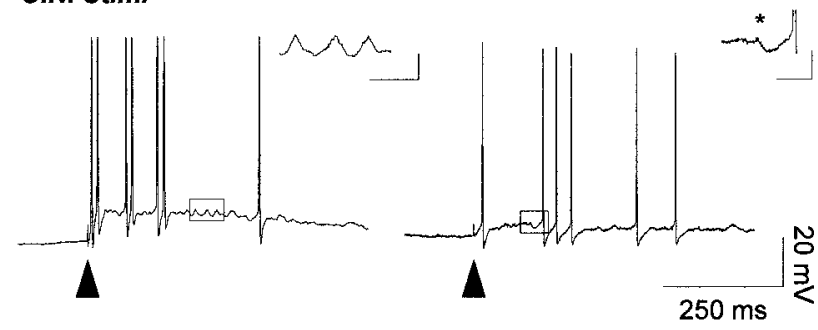

b Simulated O.N. Stim.

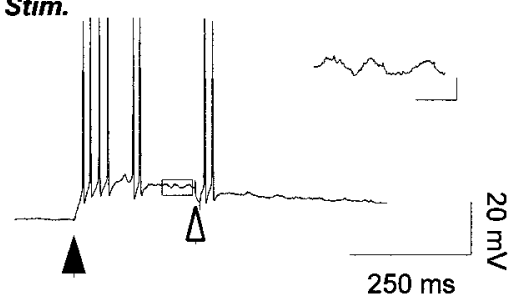

Figure 9. Electrical membrane properties of mitral cells in response to olfactory nerve stimulation. $A$, Pharmacology of olfactory nerve (O.N.)induced EPSPs. $a$, Averaged traces recorded in standard medium (STD), in presence of $100 \mu \mathrm{M} \mathrm{APV}(A P V)$, and $10 \mathrm{~mm}$ kynurenate (Kyn). b, Histograms showing the effect of APV on O.N.-evoked synaptic responses. Note that APV had small effects on EPSP amplitude (left) but consistently reduced the decay time (right). $B$, Subthreshold oscillations occur after O.N. stimulation without current injection. $B a$, Two representative synaptic responses evoked by O.N. stimulations (arrowheads) illustrate the coexistence of action potentials with subthreshold oscillatory activity. Insets zoom on the induced subthreshold oscillations (left) and a rebound spike triggered by an IPSP (noted by an asterisk on right); Calibration: $25 \mathrm{msec}, 2.5 \mathrm{mV}$. $B b$, Injection of a somatic current recorded in presence of kynurenate $(10 \mathrm{mM})$ with bicuculline $(40 \mu \mathrm{M})$ mimicking O.N.-evoked response. Note the subthreshold oscillation (inset, same scale as in $B a$ ) and the rebound spike induced by a short hyperpolarization current pulse (open arrowhead).

olfactory nerve stimulation could reset action potentials. GABAergic events elicited during olfactory nerve stimulation ( $n=4$ cells) were indeed followed by action potentials (Fig. 9Ba; the IPSP is indicated by an asterisk). This effect was also reproduced in neurons bathed with kynurenate and bicuculline in which olfactory nerve-induced depolarizations were mimicked by somatic current injection. In these conditions, rebound spikes were similarly triggered by mimicked IPSPs (Fig. 9Bb; the simulated IPSP is noted by an open arrowhead). Finally, at $32^{\circ} \mathrm{C}$, olfactory nerve stimulation evoked intracellularly recorded mitral cell responses similar to those recorded at room temperature, including subthreshold oscillations, IPSPs, and rebound spikes (data not shown; $n=5$ ).

\section{DISCUSSION}

The present study focused on subthreshold oscillations of mitral cells and their functional implications. We show that subthresh- old oscillations, ranging from 10 to $50 \mathrm{~Hz}$, are generated exclusively from a TTX-sensitive ionic conductance. The functional role of such rhythmic activity was evaluated by analyzing how mitral cells integrate synaptic potentials into action potentials. We have found that these oscillations were crucial for spike timing as well as for filtering EPSPs. We have also shown how these oscillations and spikes generated in mitral cells are temporally controlled by GABAergic synaptic inputs. Finally, we report that stimulation of the olfactory nerve, which generates excitatory inputs onto mitral cells, was sufficient to trigger stable endogenous subthreshold oscillations. We therefore propose that subthreshold oscillatory activity of the membrane potential may precisely control the timing of spiking activity and thereby provide a mechanism by which the outputs of a mitral cell population can be synchronized.

\section{Subthreshold oscillations of mitral cell membrane potentials}

Many neurons from the CNS exhibit intrinsic subthreshold fluctuations in membrane potential (Alonso and Llinás, 1989; Llinás et al., 1991; Pedroarena and Llinás, 1997). However, most of these oscillations are calcium-dependent, and few studies have described TTX-sensitive calcium-independent oscillations (Llinás, 1988). The fact that mitral cell oscillations were affected neither by changes in extracellular potassium nor by extracellular cobalt ions suggests that potassium and calcium conductances are not critical to the generation of oscillations.

Low voltage-activated calcium conductances have been described in olfactory bulb output neurons (Wang et al., 1996). Although this current might induce either subthreshold oscillations or the rhythmic generation of calcium spikes in thalamic (Pedroarena and Llinás, 1997) and neocortical (Amitai, 1994) neurons, they do not seem to be directly involved in the generation of subthreshold oscillations of mitral cells for two reasons. First, in experiments in which calcium was removed from the extracellular bath there was no modification of either the amplitude or the frequency of oscillations. Second, the hyperpolarizing current pulse protocol used to reset oscillations (Fig. 5B,C) was also insensitive to calcium removal. We propose that such oscillations are generated by a TTX-sensitive ionic conductance that operates within a range of voltages above and below spike threshold. Interestingly, the biophysical properties of the noninactivating sodium current $\left(I_{\mathrm{NaP}}\right)$ described in many excitable cells make it a suitable candidate for generating these intrinsic oscillations (for review, see Crill, 1996). In particular, its activation threshold in certain neurons was found to be approximately $-70 \mathrm{mV}$ (Parri and Crunelli, 1998), a value close to the activation of intrinsic mitral cell oscillations. Further studies of $I_{\mathrm{NaP}}$ in mitral cells will be important to understand its potential role in signal integration.

\section{Synchronization by recurrent inhibition requires subthreshold oscillations}

It has been emphasized that recurrent inhibition may generate synchronized activity in many neural networks (for review, see Jefferys et al., 1996). In the olfactory bulb, models have proposed that GABAergic inhibition exerted by inhibitory interneurons (i.e., granule cells) participates in the generation of synchronized network oscillations (for review, see Rall and Shepherd, 1968; Shepherd, 1972; Freeman, 1975). These neurons release GABA at dendrodendritic reciprocal synapses, each of which consist of an excitatory synapse directly adjacent to an inhibitory granuleto-mitral cell synapse (Price and Powell, 1970). Interestingly, this 


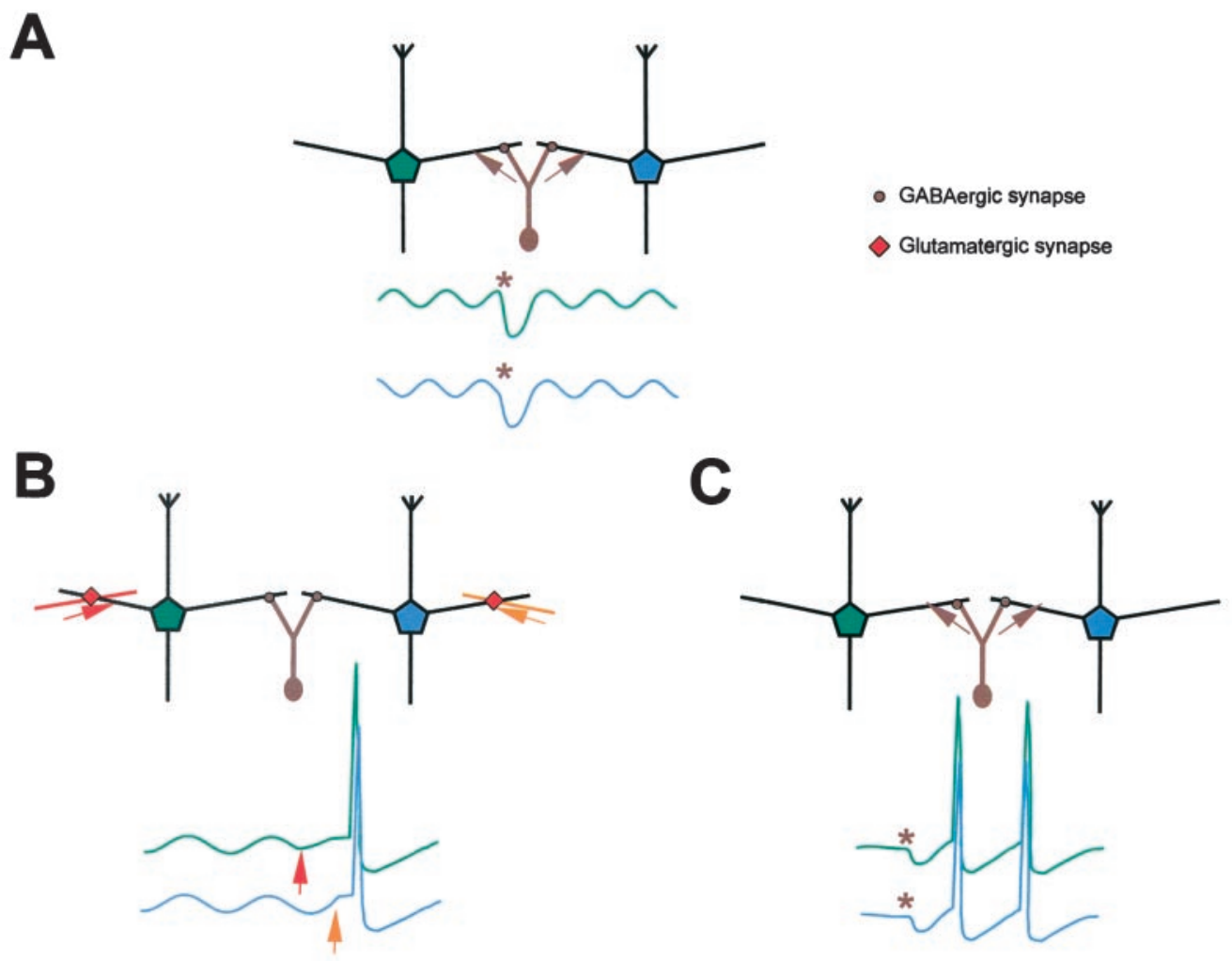

Figure 10. Proposed models for synchronization involving mitral cell intrinsic oscillations and synaptic inputs. These models require a single granule cell impinging on at least two mitral cells. $A$, Synchronization of subthreshold oscillations can result from two IPSPs in distinct mitral cells coming from the same granule cell. $B$, The synchronized oscillations can then act as a timing device for EPSP integration, thus allowing synchronization of mitral cell firing. $C$, For different membrane potentials, IPSPs can trigger rebound spikes with similar timing in two or more distinct mitral cells.

reciprocal inhibitory synapse makes the olfactory bulb an exception in the CNS and allows for fast recurrent inhibition. Our study demonstrates that interactions between GABAergic inhibition and intrinsic membrane properties is a very efficient way to precisely time spike generation. As shown in Figure $5 A$, spontaneous IPSPs were sufficient to reset the phase of endogenous subthreshold oscillations. A single granule cell which may be connected to several mitral cells could therefore effectively synchronize their intrinsic oscillations (Fig. 10 $A$ ). This possibility is supported by both physiological observations (Isaacson and Strowbridge, 1998), synaptic organization (Price and Powell, 1970), and the estimated ratio of granule cells to mitral cells of 100:1 (for review, see Mori, 1987; Shepherd and Greer, 1998). Furthermore, because granule cells are electrotonic-coupled via gap junctions (Reyher et al., 1991), granule cell activity may be synchronized, thus permitting synchronization of GABA release onto clusters of mitral cells.

Synchronization of intrinsic or synaptically induced membrane potential oscillations is a widespread phenomenon in the CNS. Synchronized oscillations have been described in several brain structures: neocortex (Steriade et al., 1996), striatum (Stern et al., 1998), and insect antennal lobe (Laurent and Davidowitz, 1994; Stopfer et al., 1997). From a theoretical point of view, it has been emphasized that synchronized subthreshold oscillations of a neural network may furnish a "context" for information processing (Buzsaki and Chrobak, 1995). However, only one report (Lampl and Yarom, 1993) supports this model. Here, as illustrated in Figure $10 B$, we propose that subthreshold oscillations of the membrane potential act as a timing device for the integration of
EPSP into spikes. Hence, whenever an EPSP impinges onto a mitral cell, the time corresponding to the highest probability for spike initiation will correspond to the peak of the next oscillation. Thus, when EPSPs occur in neighboring mitral cells with synchronized subthreshold oscillations, their triggered spikes will all occur within a narrow time window.

Because we were not able to detect any voltage-dependent amplification of the EPSPs which trigger spikes, we propose that this temporal control exerted by oscillations on spike timing is purely passive, not requiring any voltage-gated conductances. A passive mechanism would be efficient only if the EPSP kinetics had a longer duration than the oscillation period. Indeed, we observed such a relationship because the oscillation period was 50 msec, and the EPSP decay half-time constant was $61.8 \pm 10.7$ msec. This result contrasts with inferior olive neurons in which oscillations may act as a timing device for subthreshold EPSPs via voltage-dependent amplification of synaptic inputs (Lampl and Yarom, 1993).

The origin of excitatory inputs recruited by stimulation in the EPL remains unknown. One possibility supported by both anatomical (Cajal, 1911) and electrophysiological (Nicoll, 1971) data involves recurrent excitatory inputs onto secondary dendrites of mitral cells via their axon collaterals. Alternatively, mitral cells could be excited by glutamate released from the secondary dendrite of other mitral cells (Isaacson, 1999) or from excitatory centrifugal fibers. Regardless of the source of glutamate, the interactions between EPSPs and subthreshold oscillations should result from local processing, and therefore should participate in 
the formation of modules required for olfactory processing (Kauer, 1991).

Another mechanism that might allow mitral cell synchronization is the generation of a rebound spike in different mitral cells connected to a single granule cell (Fig. 10C). This mechanism could allow rapid synchronization of a cluster of mitral cells. This phenomenon has already been described in other brain structures known for their rhythmic activity, such as the thalamus (von Krosigk et al., 1993) and the hippocampus (Cobb et al., 1995). Likewise, these two phenomena depicted in Figure 10, $B$ and $C$, might also coexist in physiological conditions because stimulation of the olfactory nerve, which is known to induce firing patterns similar to those induced by odors (Freeman, 1972; Cinelli et al., 1995), triggers both subthreshold oscillations and clusters of spikes.

\section{Olfactory bulb synchronization emerges from intrinsic neuronal interactions}

Extracellular recordings have revealed that olfactory stimuli trigger the generation of network oscillations in a variety of species (for review, see Laurent, 1996b, 1997). In particular, it has been shown that mitral cell firing was phased with EEG oscillations in rodents (Freeman, 1975; Gray and Skinner, 1988). This oscillatory activity seems to be intrinsic to the olfactory bulb neural network, because olfactory or antennal nerve stimulation gives rise to EEG or LFP oscillations (Freeman, 1972) that persist after cryogenic blockade of centrifugal afferents (Gray and Skinner, 1988). These observations are consistent with our model in which synchronization emerges from intrinsic properties of the bulbar neuronal network.

\section{Distributed olfactory processing requires temporal coding}

The idea that odor information is represented in a distributed fashion within the olfactory bulb (Stewart et al., 1979; Cinelli et al., 1995) is consistent with two experimental findings. First, both olfactory sensory neurons (Getchell and Shepherd 1978; Malnic et al., 1999) and mitral cells (Wellis et al., 1989; Motokizawa, 1996; Bhalla and Bower, 1997) are very broadly tuned as they respond to a wide variety of odors. Second, it has been shown that rats with large olfactory bulb lesions can still detect as wide a variety of odors and discriminate odor mixtures as well as intact animals (Lu and Slotnick, 1994). Thus, the role of oscillations may be to allow the synchronization of populations of neurons that simultaneously process multiple odors. In the antennal lobe, pharmacological manipulations that desynchronize the activity of output cells results in a loss of discrimination between similar odors (Stopfer et al., 1997). Our study demonstrates the high degree of precision with which oscillations can time neuronal outputs, and we propose that these oscillations are crucial to olfactory processing in facilitating the synchronization of individual members of mitral cell subpopulations that may be widely distributed in the bulb.

\section{REFERENCES}

Adrian ED (1942) Olfactory reactions in the brain of the hedgehog. J Physiol (Lond) 100:459-473.

Alonso A, Llinás RR (1989) Subthreshold $\mathrm{Na}^{+}$-dependent theta-like rhythmicity in stellate cells of entorhinal cortex layer II. Nature 342:175-177.

Amitai Y (1994) Membrane potential oscillations underlying firing patterns in neocortical neurons. Neuroscience 63:151-161.

Bhalla US, Bower JM (1997) Multiday recordings from olfactory bulb neurons in awake freely moving rats: spatially and temporally orga- nized variability in odorant response properties. J Comput Neurosci 4:221-256.

Buzsaki G, Chrobak JJ (1995) Temporal structure in spatially organized neuronal ensembles: a role for interneuronal networks. Curr Opin Neurobiol 5:504-510.

Cajal RY (1911) Histologie du système nerveux de l'homme et des vertébrés. Paris: Maloine.

Chen WR, Shepherd GM (1997) Membrane and synaptic properties of mitral cells in slices of rat olfactory bulb. Brain Res 745:189-196.

Cinelli AR, Hamilton KA, Kauer JS (1995) Salamander olfactory bulb neuronal activity observed by video rate, voltage-sensitive dye imaging. III. Spatial and temporal properties of responses evoked by odorant stimulation. J Neurophysiol 73:2053-2071.

Cobb SR, Buhl EH, Halasy K, Paulsen O, Somogyi P (1995) Synchronization of neuronal activity in hippocampus by individual GABAergic interneurons. Nature 378:75-78.

Connors BW, Amitai Y (1997) Making waves in the neocortex. Neuron 18:347-349.

Crill WE (1996) Persistent sodium current in mammalian central neurons. Annu Rev Physiol 58:349-362.

Delaney KR, Hall BJ (1996) An in vitro preparation of frog nose and brain for the study of odour-evoked oscillatory activity. J Neurosci Methods 68:193-202.

Ennis M, Zimmer LA, Shipley MT (1996) Olfactory nerve stimulation activates rat mitral cells via NMDA and non-NMDA receptors in vitro. NeuroReport 7:989-992.

Freeman W (1972) Spatial divergence and temporal dispersion in primary olfactory nerve of the cat. J Neurophysiol 35:733-744.

Freeman WJ (1975) Mass action in the nervous system. New York: Academic.

Gelperin A, Tank DW (1990) odour-modulated collective network oscillations of olfactory interneurons in a terrestrial mollusc. Nature 345:437-440.

Getchell TV, Shepherd GM (1978) Responses of olfactory receptor cells to step pulses of odour at different concentrations in the salamander. J Physiol (Lond) 282:521-540.

Gray CM (1994) Synchronous oscillations in neuronal systems: mechanisms and functions. J Comput Neurosci 1:11-38.

Gray CM, Skinner JE (1988) Centrifugal regulation of neuronal activity in the olfactory bulb of the waking rabbit as revealed by reversible cryogenic blockade. Exp Brain Res 69:378-386.

Gutfreund Y, Yarom Y, Segev I (1995) Subthreshold oscillations and resonant frequency in guinea-pig cortical neurons: physiology and modelling. J Physiol (Lond) 483:621-640.

Haag J, Borst A (1996) Amplification of high-frequency synaptic inputs by active dendritic membrane processes. Nature 379:639-641.

Häusser M, Clark BA (1997) Tonic synaptic inhibition modulates neuronal output pattern and spatiotemporal synaptic integration. Neuron 19:665-678.

Hille B (1992) Ionic channels of excitable membranes, Ed 2. Sunderland, MA: Sinauer.

Isaacson JS (1999) Glutamate spillover mediates excitatory transmission in the rat olfactory bulb. Neuron 23:377-384.

Isaacson JS, Strowbridge BW (1998) Olfactory reciprocal synapses: dendritic signaling in the CNS. Neuron 20:749-761.

Jefferys JG, Traub RD, Whittington MA (1996) Neuronal networks for induced "40 Hz" rhythms. Trends Neurosci 19:202-208.

Kauer JS (1991) Contributions of topography and parallel processing to odor coding in the vertebrate olfactory pathway. Trends Neurosci 14:79-85.

Keller A, Yagodin S, Aroniadou-Anderjaska V, Zimmer LA, Ennis M, Sheppard Jr NF, Shipley MT (1998) Functional organization of rat olfactory bulb glomeruli revealed by optical imaging. J Neurosci 18:2602-2612.

König P, Engel AK, Singer W (1996) Integrator or coincidence detector? The role of the cortical neuron revisited. Trends Neurosci 19:130-137.

Lampl I, Yarom Y (1993) Subthreshold oscillations of the membrane potential: a functional synchronizing and timing device. J Neurophysiol 70:2181-2186.

Larkum ME, Zhu JJ, Sakmann B (1999) A new cellular mechanism for coupling inputs arriving at different cortical layers. Nature 398:338-341.

Laurent G, Davidowitz H (1994) Encoding of olfactory information with oscillating neural assemblies. Science 265:1872-1875. 
Laurent G (1996a) Odor images and tunes. Neuron 16:473-476.

Laurent G (1996b) Dynamical representation of odors by oscillating and evolving neural assemblies. Trends Neurosci 19:489-496.

Laurent G (1997) Olfactory processing: maps, time and codes. Curr Opin Neurobiol 7:547-553.

Laurent G, Wehr M, Davidowitz H (1996) Temporal representations of odors in an olfactory network. J Neurosci 16:3837-3847.

Llinás R (1988) The intrinsic electrophysiological properties of mammalian neurons: insights into central nervous system function. Science 242:1654-1664.

Llinás R, Grace AA, Yarom Y (1991) In vitro neurons in mammalian cortical layer 4 exhibit intrinsic oscillatory activity in the $10-$ to $50-\mathrm{Hz}$ frequency range. Proc Natl Acad Sci USA 88:897-901.

Lu XC, Slotnick BM (1994) Recognition of propionic acid vapor after removal of the olfactory bulb area associated with high 2-DG uptake. Brain Res 639:26-32.

MacLeod K, Laurent G (1996) Distinct mechanisms for synchronization and temporal patterning of odor-encoding neural assemblies. Science 274:976-979.

Malnic B, Hirono J, Sato T, Buck LB (1999) Combinatorial receptor codes for odors. Cell 96:713-723.

Mori K (1987) Membrane and synaptic properties of identified neurons in the olfactory bulb. Prog Neurobiol 29:275-320.

Motokizawa F (1996) Odor representation and discrimination in mitral/ tufted cells of the rat olfactory bulb. Exp Brain Res 112:24-34.

Nicoll RA (1971) Recurrent excitation of secondary olfactory neurons: a possible mechanism for signal amplification. Science 171:824-826.

Parker AJ, Newsome WT (1998) Sense and the single neuron: probing the physiology of perception. Annu Rev Neurosci 21:227-277.

Parri HR, Crunelli V (1998) Sodium current in rat and cat thalamocortical neurons: role of a non-inactivating component in tonic and burst firing. J Neurosci 18:854-867.

Pedroarena C, Llinás R (1997) Dendritic calcium conductances generate high-frequency oscillation in thalamocortical neurons. Proc Natl Acad Sci USA 94:724-728.

Perkel DH, Gerstein GL, Moore GP (1967) Neuronal spike trains and stochastic point processes. I. The single spike train. Biophys J 4:391-418.

Price JL, Powell TPS (1970) The morphology of granule cells within the olfactory bulb. J Cell Sci 7:91-123.

Rall W, Shepherd GM (1968) Theoretical reconstruction of field potentials and dendrodrendritic synaptic interactions in olfactory bulb. J Neurophysiol 31:884-915.

Reyher C K, Lubke J, Larsen WJ, Hendrix GM, Shipley MT, Baumgarten HG (1991) Olfactory bulb granule cell aggregates: morphological ev- idence for interperikaryal electrotonic coupling via gap junctions. J Neurosci 11:1485-1495.

Riehle A, Grun S, Diesmann M, Aertsen A (1997) Spike synchronization and rate modulation differentially involved in motor cortical function. Science 278:1950-1953.

Rieke F, Warland D, de Ruyter van Steveninck R, Bialek W (1997) Spike: Exploring the neural code. Cambridge, MA: MIT.

Ritz R, Sejnowski TJ (1997) Synchronous oscillatory activity in sensory systems: new vistas on mechanisms. Curr Opin Neurobiol 7:536-546.

Satou M, Ueda K (1978) Synchronized rhythmic discharges of the secondary olfactory neurons in carp. Brain Res 158:313-329.

Shepherd GM (1972) Synaptic organization of the mammalian olfactory bulb. Physiol Rev 52:864-917.

Shepherd GM, Greer CA (1998) Olfactory bulb. In: The synaptic organization of the brain, Ed 4 (Shepherd GM, ed), pp 159-203. New-York: Oxford UP.

Silva LR, Amitai Y, Connors BW (1991) Intrinsic oscillations of neocortex generated by layer 5 pyramidal neurons. Science 251:432-435.

Spencer WA, Kandel ER (1961) Electrophysiology of hippocampal neurons. IV Fast prepotentials. J Neurophysiol 24:272-285.

Steriade M, Amzica F, Contreras D (1996) Synchronization of fast $(30-40 \mathrm{~Hz})$ spontaneous cortical rhythms during brain activation. J Neurosci 16:392-417.

Stern EA, Jaeger D, Wilson CJ (1998) Membrane potential synchrony of simultaneously recorded striatal spiny neurons in vivo. Nature 394:475-478

Stewart WB, Kauer JS, Shepherd GM (1979) Functional organization of rat olfactory bulb analysed by the 2-deoxyglucose method. J Comp Neurol 185:715-734.

Stopfer M, Bhagavan S, Smith B, Laurent G (1997) Impaired odour discrimination on desynchronization of odour-encoding neural assemblies. Nature 390:70-74.

Stuart G, Sakmann B (1995) Amplification of EPSPs by axosomatic sodium channels in neocortical pyramidal neurons. Neuron 15:1065-1076.

Tank DW, Gelperin A, Kleinfeld D (1994) Odors, oscillations, and waves: does it all compute? Science 265:1819-1820.

von Krosigk M, Bal T, McCormick DA (1993) Cellular mechanisms of a synchronized oscillation in the thalamus. Science 261:361-364.

Wang XY, McKenzie JS, Kemm RE (1996) Whole cell calcium currents in acutely isolated olfactory bulb output neurons of the rat. J Neurophysiol 75:1138-1151.

Wellis DP, Scott JW, Harrison TA (1989) Discrimination among odorants by single neurons of the rat olfactory bulb. J Neurophysiol 61: 1161-1177. 